\title{
In eigener Sache: Hot Topics als neue Form wissenschaftlicher Artikel in der Unterrichtswissenschaft
}

\section{Cornelia Gräsel • Ilonca Hardy • Silke Hertel • Thilo Kleickmann • Knut Neumann • Anna-Katharina Praetorius • Alexander Renkl • Joachim Wirth}

Aufgrund der dynamischen Entwicklung in unserem Forschungsfeld haben nicht nur Bildungspolitiker*innen und Praktiker*innen Schwierigkeiten, sich ein fundiertes Urteil über aktuelle Bildungsfragen zu bilden. Auch den Forscher*innen selbst fällt dies in Gebieten schwer, die nicht zu ihrem eigenen Themenspektrum gehören.

Daher haben die Herausgeber*innen der Unterrichtswissenschaft beschlossen, eine neue Form wissenschaftlicher Artikel in das Heft aufzunehmen, nämlich Texte zu Hot Topics. Die Herausgeber*innen bitten Expert*innen, sich in einem Text zu einem aktuellen, gesellschaftlich relevanten und kontroversen Bildungsthema zu positionieren und ihre Position vor dem Hintergrund des aktuellen Forschungsstands zu

C. Gräsel $(\bowtie)$

Bergische Universität Wuppertal, Wuppertal, Deutschland

E-Mail: graesel@uni-wuppertal.de

I. Hardy

Universität Frankfurt a. M., Frankfurt a. M., Deutschland

S. Hertel

Universität Heidelberg, Heidelberg, Deutschland

T. Kleickmann

Universität Kiel, Kiel, Deutschland

K. Neumann

Leibniz-Institut für die Pädagogik der Naturwissenschaften und Mathematik (IPN), Kiel, Deutschland

A.-K. Praetorius

Universität Zürich, Zürich, Schweiz

A. Renkl

Universität Freiburg, Freiburg, Deutschland

J. Wirth

Ruhr-Universität Bochum, Bochum, Deutschland 
begründen. Die Hot Topics haben nicht den Anspruch eines systematischen Reviews oder eines Kurzreviews. Sie sollen vielmehr wissenschaftlich fundierte Standpunkte darstellen und immer auch reflektieren, welche Konsequenzen für die aus dem aktuellen Forschungsstand gezogen werden können.

Der aktuelle Beitrag von Thamar Voss und Jörg Wittwer ${ }^{1}$ (in diesem Heft) zum Thema „Unterricht in Zeiten von Corona: Ein Blick auf die Herausforderungen aus der Sicht von Unterrichts- und Lehr-Lern-Forschung“ ist nicht der erste Beitrag dieser Art in der Unterrichtswissenschaft. Bereits letztes Jahr erschien - noch ohne Einführung - ein erster entsprechender Text von Scheiter und Lachner (2019) zum Thema „DigitalPakt - was nun?“. Die Serie der Hot Topics wird, je nachdem, wann und wie Themen wichtig werden, in unregelmäßigen Abständen fortgesetzt. Damit knüpft die Unterrichtswissenschaft mit diesem neuen Format an ihre Tradition an, wissenschaftliche Ergebnisse in verschiedenen Communities der Bildungsforschung und der Bildungspraxis zu verbreiten und zur Diskussion darüber anzuregen. Gleichzeitig wollen wir als Herausgeber*innen einen Beitrag dazu leisten, dass aktuelle und kontroverse Bildungsfragen stärker unter Berücksichtigung wissenschaftlich begründeten Wissens diskutiert werden.

Funding Open Access funding enabled and organized by Projekt DEAL.

Open Access Dieser Artikel wird unter der Creative Commons Namensnennung 4.0 International Lizenz veröffentlicht, welche die Nutzung, Vervielfältigung, Bearbeitung, Verbreitung und Wiedergabe in jeglichem Medium und Format erlaubt, sofern Sie den/die ursprünglichen Autor(en) und die Quelle ordnungsgemäß nennen, einen Link zur Creative Commons Lizenz beifügen und angeben, ob Änderungen vorgenommen wurden.

Die in diesem Artikel enthaltenen Bilder und sonstiges Drittmaterial unterliegen ebenfalls der genannten Creative Commons Lizenz, sofern sich aus der Abbildungslegende nichts anderes ergibt. Sofern das betreffende Material nicht unter der genannten Creative Commons Lizenz steht und die betreffende Handlung nicht nach gesetzlichen Vorschriften erlaubt ist, ist für die oben aufgeführten Weiterverwendungen des Materials die Einwilligung des jeweiligen Rechteinhabers einzuholen.

Weitere Details zur Lizenz entnehmen Sie bitte der Lizenzinformation auf http://creativecommons.org/ licenses/by/4.0/deed.de.

\section{Literatur}

Scheiter, K., \& Lachner, A. (2019). DigitalPakt - was nun? Eine Positionierung aus Sicht der Lehr-Lernforschung. Unterrichtswissenschaft, 47(4), 547-564.

\footnotetext{
1 Geteilte Erstautorenschaft.
} 